\title{
LIE POINT TRANSFORMATIONS ADMITTED BY E. WITTEN EQUATIONS
}

T. HALLAP, K. KIIRANEN. E. WITTENI VORRANDITE POOLT LUBATUD LIE PUNKTTEISENDUSED

T. ХАЛЛАП, К. КИИРАНЕН. ТОЧЕЧНЫЕ ПРЕОБРАЗОВАНИЯ ЛИ, ДОПУСКАЕМЫЕ УРАВНЕНИЯМИ Э. ВИТТЕНА

(Presented by H. Keres)

The Yang-Mills equations are nonlinear second order partial differential equations for 12 scalar functions $\stackrel{a}{A_{\mu}}(x) \quad\left[{ }^{1}\right]$ :

$$
\partial_{\mu} \stackrel{a}{F}_{\mu v}+\varepsilon^{a b c} \stackrel{b}{A_{\mu}} \stackrel{c}{F}_{\mu \nu}=0,
$$

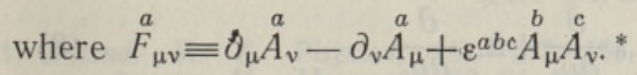

If the functions $\stackrel{a}{A_{\mu}}(x)$ satisfy the so-called self-dual Yang-Mills equations

$$
\stackrel{a}{F_{\mu \nu}}-\frac{1}{2} \varepsilon_{\mu \nu \alpha \beta} \stackrel{a}{F} \alpha \beta=0,
$$

then they satisfy equations (1) as well. Note that the system of nonlinear first order partial differential equations (2) contains 9 equations and is thereby an underdeterminate system.

E. Witten has searched for possible solutions for equations (2) in cylindrical form $\left[{ }^{2}\right]$ :

$$
\begin{aligned}
& \stackrel{a}{A_{0}}=\frac{A_{0} x_{a}}{r}, \\
& \stackrel{a}{A_{i}}=\frac{1+\varphi_{2}}{r^{2}} \varepsilon_{i a k} x_{k}+\frac{\varphi_{1}}{r^{3}}\left(\delta_{i a} r^{2}-x_{i} x_{a}\right)+A_{1} \frac{x_{1} x_{a}}{r^{2}},
\end{aligned}
$$

where scalar functions $A_{0}, A_{1}, \varphi_{1}, \varphi_{2}$ depend only on two variables $t$ and $r$, where $r^{2} \equiv x_{k} x_{k}$. This ansatz gives the model which is very similar to the Abelian Higgs model in space with constant negative curvature.

When we take

$$
\begin{array}{ll}
A_{0}=u_{3}+\frac{1}{r}, & A_{1}=u_{4}, \\
\varphi_{1}=r u_{1}, & \varphi_{2}=-r u_{2}
\end{array}
$$

* Here the sum convention is observed. The Greek indices take values from 1 to 4 , the Latin ones - from 1 to 3 . Only $\mathrm{SU}(2)$ gauge group is considered and constant $g \equiv 1$ for simplicity's sake. 
and note $t \equiv x_{0}, r \equiv x_{1}$ and $\partial_{0} \equiv \frac{\partial}{\partial t}, \partial_{1} \equiv \frac{\partial}{\partial r}$, then we get the following system of equations for functions $u_{1}, u_{2}, u_{3}, u_{4}$ :

$$
\begin{aligned}
& u_{, 0}^{1}+u_{, 1}^{2}+u^{1} u^{4}-u^{2} u^{3}=0 \\
& u_{, 0}^{2}-u_{, 1}^{1}+u^{1} u^{3}+u^{2} u^{4}=0 \\
& u_{, 0}^{4}-u_{, 1}^{3}+u^{1} u^{1}+u^{2} u^{2}=0 .
\end{aligned}
$$

We call these E. Witten equations. Such equations arise also in the theory of minimal surfaces as Gauss-Codazzi equations [ $\left.{ }^{3}\right]$. In fact, in this case there is one more equation:

$$
u_{, 0}^{3}+u_{, 1}^{4}=0 \quad \text { or } \quad \partial_{0} A_{0}+\partial_{1} A_{1}=0 .
$$

E. Witten uses this equation in searching for possible solutions of system (5). The system of equations (5) - (6) is a determinate one.

The results of the calculations following method [ $\left.{ }^{4}\right]$ indicate that the generators of Lie point transformations group admitted by the equations (5) form an infinite-dimensional algebra $L_{\infty}$ :

$$
\begin{aligned}
X & =f \frac{\partial}{\partial x_{0}}+g \frac{\partial}{\partial x_{1}}-u_{1} f, 0 \frac{\partial}{\partial u_{1}}-u_{2} g, \frac{\partial}{\partial u_{2}}+ \\
& +\left(u_{4} f_{, 1}-u_{3} f_{, 0}-f_{, 01}\right) \frac{\partial}{\partial u_{3}}+\left(u_{3} g_{, 0}-u_{4} g_{, 1}+g, g_{, 1}\right) \frac{\partial}{\partial u_{4}}, \\
Y & =h\left(u_{2} \frac{\partial}{\partial u_{1}}-u_{1} \frac{\partial}{\partial u_{2}}\right)+h, 0 \frac{\partial}{\partial u_{4}}+h, 1 \frac{\partial}{\partial u_{4}},
\end{aligned}
$$

where $f_{, 1}+g_{, 0}=0, f_{, 0}=g_{, 1}=0$ and $h\left(x_{0}, x_{1}\right)$ is an arbitrary function. If we take into account equation (6) as well, then

$$
h, 00+h,{ }_{11}=0 \text {. }
$$

The generators of Lie point transformations group for equations (1) and (2) were computed in [ $\left.{ }^{5}\right]$. The result obtained was the algebra $L_{\infty}=$ $=L_{15} \oplus L^{0}$ in both cases where $L_{15}$ is the algebra of the conformal group and $L^{0}$ contains the gauge transformations:

$$
\begin{aligned}
& X=\xi^{\mu} \frac{\partial}{\partial x^{\mu}}-\xi_{, \mu}^{v} \stackrel{a}{A_{\nu}} \frac{\partial}{\partial \stackrel{a}{A_{\mu}}}, \\
& Y=\stackrel{\zeta}{\mu}_{\mu} \frac{\partial}{\partial \stackrel{a}{A}_{\mu}}
\end{aligned}
$$

where

$$
\xi_{\mu}(x)=k_{\mu}+d \cdot x_{\mu}+a_{\mu v} x_{v}+2 c_{v} x_{v} x_{\mu}-c_{\mu} x_{v} x_{v}
$$

is the Killing vector and

$$
\stackrel{a}{\zeta}_{\mu}(x, A)=\stackrel{a}{\theta}, \mu-\stackrel{b}{a}^{a b c} \stackrel{\oplus}{A}_{\mu} .
$$

$\stackrel{a}{\theta}(x)$ denote arbitrary functions of $x_{\mu}$ and $d, k_{\mu}, c_{\mu}, a_{\mu v}=-a_{v \mu}$ are arbitrary constants.

In the two-dimensional case $(n=2) f \equiv \xi^{0}$ and $g \equiv \xi^{1}$ from (7)-(8) are arbitrary functions of variables $x_{0} \pm i x_{1}$, whereas in the general $n>2$ (here $n=4$ ) the functions $\xi^{\mu}(x)$ are second order polynoms. 
The generator $Y$ describes the gauge transformations in both cases and it is the ideal of the algebra since

$$
[X, Y]=Y \text {. }
$$

However, when the functions $\stackrel{a}{A_{\mu}}$ satisfy the Lorentz gauge condition

$$
\partial_{\mu} \stackrel{a}{A_{\mu}}=0,
$$

then in transformations $(9)-(10)$

$$
\stackrel{a}{\theta}, \mu=0 \quad \text { and } \quad \stackrel{a}{A} c_{\mu}=0 .
$$

Consequently, the special conformal transformation will disappear and in place of gauge transformations only rotations $\mathrm{SO}(3) \simeq \mathrm{SU}(2)$ in isotopic space will remain:

$$
Y_{a b}=\stackrel{a}{A_{\mu}} \frac{\partial}{\partial \stackrel{b}{A}_{\mu}}-\stackrel{b}{A_{\mu}} \frac{\partial}{\partial A_{\mu}} .
$$

In this case the finite algebra $L_{14}=L_{11} \oplus L_{3}\left[{ }^{6}\right]$. The authors are grateful to $M$. Kõiv for discussions.

\section{REFERENCES}

1. Prasad, M. K. Physica, 1D, 167-191 (1980); В кн.: Геометрические идеи в физике. М., «Наука», 1983, 64-96.

2. Witten, E. Phys. Rev. Lett., 38, № 3, 121-124 (1977).

3. Comtet, A. Phys. Rev., D18, № 10, 3890-3892 (1978).

4. Овсянников Л. В. Лекции по теории групповых свойств дифференциальных уравнений. Новосибирск, «Наука, 1966.

5. Schwarz, F. Lett. Math. Phys., 6, № 5, 355-359 (1982).

6. Rosenhaus, V., Kiiranen, K. ENSV TA Toim. Füüs. Matem., 31, № 3, 304-312 (1982).

Tartu State University

Received

March 20, 1987 